\title{
Loss of Monoacylglycerol 0-Acyltransferase 2 can be Compensated for by Diacylglycerol 0- Acyltransferases 1 and 2 in High-Fat Diet-Induced Obesity and Mammary Cancer Development
}

\author{
Yan Mei \\ Sun Yat-sen University Cancer Center \\ Jing Wang \\ Sun Yat-sen University Cancer Center \\ Jia-Bin Lu \\ Sun Yat-sen University Cancer Center \\ Guan-Ming Lu \\ Youjiang Medical University for Nationalities \\ Li-Xia Peng \\ Sun Yat-sen University Cancer Center \\ Yan-Hong Lang \\ Sun Yat-sen University Cancer Center \\ Li-Sheng Zheng \\ Sun Yat-sen University Cancer Center \\ Bi-Jun Huang \\ Sun Yat-sen University Cancer Center \\ Yan-Xia Shi \\ Sun Yat-sen University Cancer Center \\ Chaonan Qian ( $\nabla$ qianchn@sysucc.org.cn ) \\ Sun Yat-sen University Cancer Center https://orcid.org/0000-0002-7233-8831
}

Primary research

Keywords: Mogat2 depletion, MMTV-PyMT, mammary tumor, Dgat1, Dgat2.

Posted Date: October 5th, 2021

DOI: https://doi.org/10.21203/rs.3.rs-398464/v2

License: (c) (i) This work is licensed under a Creative Commons Attribution 4.0 International License.

Read Full License 
Page 2/17 


\section{Abstract}

Background: Dietary fat absorption involves the re-esterification of digested triacylglycerol in the enterocytes, it is a biological process catalyzed by monoacylglycerol 0-acyltransferase 2 (MOGAT2, aka MGAT2), which is highly expressed in the small intestine. A previous study showed that the loss of the Mogat2 gene can prevent high-fat diet-induced obesity in mice. Obesity is associated with an increased risk of several types of cancer including postmenopausal breast cancer.

Methods: We collected 147 patients with triple negative breast adenocarcinoma to explore the relationship between the expression of MOGAT2 and patient overall survival. And we generated a Mogat2-deficient mouse mammary tumor model by crossing Mogat2-deficient mice with MMTV-PyMT mice to examine the effect of losing MOGAT2 in vivo.

Results: Our founding suggest that obesity was induced by a relatively high-fat diet (37\% of calories from fat) in the mice with or without Mogat2 knockout. Mammary tumor development was deteriorated by a relatively high-fat diet regardless of Mogat2 deficiency. As a compensation mechanism, upregulation of diacylglycerol 0-acyltransferases 1 and 2 (Dgat1 and Dgat2) in the Mogat2 deficient mice was found.

Conclusions: Elevated expression of MOGAT2 in triple negative breast adenocarcinoma predicts poorer patient overall survival. With the compensation of Dgat1 and Dgat2, Mogat2 deficiency alone cannot prevent fat diet-induced obesity, nor prevent mammary tumor development in a mouse model.

\section{Background}

Breast cancer is the most commonly diagnosed cancer and the second most common cause of cancerinduced death in women worldwide (1). Over 600,000 premenopausal and 1.4 million postmenopausal breast cancer cases were diagnosed around the world in 2018 (2). Relatedly, obesity poses an increased risk in developing breast cancer, particularly in postmenopausal women (1). Sustained weight loss can significantly reduce breast cancer risk (3). However, the impact of genetically preventing obesity on breast cancer risk is unclear.

Triacylglycerols, the bulk of dietary fat, are absorbed in the intestine and readily stored as body fat (4). The hydrolysis of triacylglycerols to 2-monoacylglycerol and fatty acids are primarily catalyzed by pancreatic lipase in the intestinal lumen, where these hydrolysis products are taken up by enterocytes and resynthesized into triacylglycerols via the monoacylglycerol pathway (5). The monoacylglycerol pathway is a process catalyzed by the acyl-CoA: monoacylglycerol acyltransferase (MOGAT) family which includes MOGAT1, MOGAT2, and MOGAT3, and accounts for $70-80 \%$ of triglyceride re-synthesis in the intestine $(6,7)$. Another pathway involved in triglyceride synthesis is the glycerol 3-phosphate pathway (8). Among the three MOGAT enzymes, only MOGAT2 (also known as MGAT2) is highly expressed in the intestine of both mice and humans (9-12). 
It has been reported that a deficiency in MOGAT2 protects mice from obesity and associated metabolic disorders induced by a high fat diet $(13,14)$. MOGAT2 deficiency also protects genetically obese Agouti mice from excess weight gain on a chow diet (4). Diacylglycerol (DAG) is usually acylated by the acyl coenzyme A: diacylglycerol acyltransferase (DGAT) to re-synthesize triacylglycerol (TAG). Importantly, enzymes DGAT1 (15) and DGAT2 have been shown to acylate MAG to form DAG, and MOGAT2 and MOGAT3 have the capacity to acylate DAG to form TAG $(16,17)$.

To elucidate the role of MOGAT2 in breast cancer progression, especially under obese conditions, we evaluated the prognostic value of MOGAT2 expression in triple negative breast adenocarcinoma, and generated a MOGAT2-deficient mouse mammary tumor model by crossing MOGAT2-deficient mice with MMTV-PyMT mice.

\section{Methods}

\section{Mice and animal care}

FVB/N-Tg (MMTV-PyMT) 634Mul mice and B6.129S4-Mogat2 ${ }^{\mathrm{tm} 1 \mathrm{Far} / \mathrm{J}}\left(\mathrm{MOGAT2}^{-/-}\right)$were purchased from the Jackson Laboratory (Bar Harbor, ME, USA). Mice were housed under specific pathogen-free conditions in the Animal Center of Guangdong Pharmaceutical University. All animal experiments were performed in accordance with institutional guidelines and were approved by Sun Yat-sen University Cancer Center's Institutional Animal Care and Usage Committee, with the ethical approval number L102012017000G. The control group were fed by a normal diet consisting of $4 \%$ fat, $65 \%$ carbohydrates and $20 \%$ protein (weight ratio). And $10 \%$ of the total calories were from fat. The experimental group were fed by a relatively high-fat diet consisting of $20 \%$ sugar, $15 \%$ lard, $1.2 \%$ cholesterol, $0.2 \%$ sodium cholate, $10 \%$ casein, $0.6 \%$ calcium hydrogen phosphate, $0.4 \%$ stone powder, $0.4 \%$ premix, and a $52.2 \%$ basal diet (weight ratio). And $37 \%$ of the total calories were from fat. The normal diet and the relatively high fat diet were purchased from the Experimental Medicine Animals Center (Foshan, China).

MMTV-PyMT (PyMT) male animals were bred with Mogat2 $^{-1-}$ females to produce Mogat2 $^{+/-}{ }^{-}$PyMT male and Mogat2 $^{+/-}$female F1 hybrids. F1 Mogat2 ${ }^{+/-}$PyMT male mice were then mated with Mogat2 $2^{+-}$ female mice to produce F2 Mogat2 ${ }^{+/}$PyMT and Mogat2 ${ }^{-1-}$ PyMT female mice.

All animals were killed by inhalation of carbon dioxide. Putting the animals into a closed transparent container, carbon dioxide is injected into the container at a rate of $30 \%$ per minute, until the animals die.

\section{Histological examination of murine tissues}

Whole-mount carmine alum staining was performed according to the standard protocol (18). The inguinal mammary glands from 5-week-old F2 Mogat2 ${ }^{+/}$PyMT and Mogat2 ${ }^{-1-}$ PyMT female mice were fixed overnight in Carnoy's solution (75\% absolute ethanol [EtOH] and $25 \%$ glacial acetic acid [Sigma-Aldrich]). Following fixation, the glands were washed with $70 \%$ ethanol for 30 mins, gradually rinsed in water and stained with carmine alum (Sigma-Aldrich) for at least two days. To examine the lung metastasis foci, 
tissues were fixed in Bouin's solution (Sigma-Aldrich) for two days, and the number of metastatic lesions larger than $0.5 \mathrm{~mm}$ in diameter were counted macroscopically.

\section{RT-PCR}

At 98 days old, the F2 Mogat2 $^{+/+}$PyMT and Mogat2 $^{-/-}$PyMT female mice were euthanized for tissue harvesting. Mammary tumors, stomach, small intestine and colorectal tissues were collected and stored in RNAstore reagent (TIANGEN) for RNA isolation.

Total RNA was extracted from mice tissues using Trizol reagent (Invitrogen, CA, USA) and subjected to reverse transcription using a PrimeScript ${ }^{\text {TM }}$ II 1st Strand cDNA Synthesis Kit (Takara), followed by reactions using the Roche LightCycler 480 System (Roche). Beta-actin served as the normalization genes for these studies. The relative expression levels of the target genes were calculated using the $2-\Delta \Delta \mathrm{Ct}$ method. The qPCR primers were obtained from PrimerBank (19).

\section{Human tissue specimens}

A total of 147 paraffin-embedded triple negative primary breast cancer tissues, diagnosed between 2005 and 2009, were retrieved from the Department of Pathology at Sun Yat-sen University Cancer Center (SYSUCC). All human tissue samples were obtained with patient consent and the approval of the Institutional Clinical Ethics Review Board at SYSUCC. Median follow-up time for all patients was 102 months.

The IHC scores for MOGAT2 (Cat\#ab62526, Abcam) in the breast cancer tissues were calculated by two independent pathologists. For each tumor, two scores were given. One for the percentage of positively stained tumor cells, and another for staining intensity. The percentage of positive cells were categorized as: no staining $=0,1-10 \%$ of stained cells $=1,11-50 \%=2,51-80 \%=3$ and $81-100 \%=4$. Cytoplasmic staining intensity was categorized as: no staining $=0$, weak staining $=1$, moderate staining $=2$ and strong staining $=3$. The proportion and intensity were then multiplied to produce a total score ranging from 0 to 12. A cut-off value of 4 was the optimal balance between sensitivity and specificity with the patients divided into high-level $(n=51)$ and low-level $(n=96)$ groups.

\section{Statistical analysis}

Statistical analyses were performed using GraphPad Prism v7.0 software and the data presented as mean \pm SEM. The correlations between MOGAT2 expression and overall survival, mice lacking palpable tumors and the mice's age were analyzed with Kaplan-Meier survival curves and the log-rank test. The differences between the groups were compared using Student's t-test, and ANOVA for multiple comparisons. P-values were used to denote statistical significance.

\section{Results}




\section{Elevated MOGAT2 expression predicts poorer prognosis in breast cancer patients}

IHC staining showed that MOGAT2 was localized in the cytoplasm of breast cancer cells (Fig. 1A). Survival analysis revealed that elevated expression of MOGAT2 correlated with poorer overall survival in patients with triple negative breast adenocarcinoma (Fig. 1B). This suggests that depletion of MOGAT2 might be beneficial to the host in animal models.

\section{A relatively high fat diet promotes obesity regardless of MOGAT2 deficiency}

Previous studies $(13,14,20)$ reported that MOGAT2 ablation protected mice from high-fat diet induced obesity ( $60 \%$ of calories from fat). In our study, in the mice fed with a relatively high fat diet ( $37 \%$ calories from fat) between 5 and 12 weeks old, obesity was observed regardless of MOGAT2 deficiency (Fig. 2).

\section{MOGAT2 depletion does not inhibit mammary tumorigenesis or metastasis}

To determine whether loss of MOGAT2 in MMTV-PyMT mice affects the development of multifocal dysplastic lesions, we collected the right fourth (inguinal) mammary gland from virgin female Mogat2//+PyMT and Mogat2 ${ }^{-/}$PyMT mice at exactly 35 days old, fixed these tissues in Carnoy's solution, and stained them with carmine dye for at least two days. At 35 days old, Mogat2 ${ }^{+/+}$PyMT and Mogat2 ${ }^{-1}$ PyMT mice both exhibited small hyperplastic focal lesions in the older portions of the mammary ductal tree (Fig. 3A). However, there was no significant difference in the frequency or size of these benign lesions in the absence of MOGAT2.

The effect of MOGAT2 ablation on tumor onset was monitored by weekly palpation as shown in Fig. 3B. No statistical significant difference was observed between the two groups. All female Mogat ${ }^{+/+}{ }^{+}$PyMT and Mogat2 ${ }^{-/}$PyMT mice developed mammary tumors within 77 days.

To assess MOGAT2's depletion effect on mammary tumor progression, the number of tumor-affected mammary glands and total tumor volume were monitored by weekly palpation. The results showed there was no statistically significant difference between the two groups (Fig. 4A and 4B).

The effect of MOGAT2 ablation on the number of metastases was also determined. Female Mogat2 ${ }^{+/+}$PyMT and Mogat2 ${ }^{-/}$PyMT mice were euthanized at 16 weeks old, the lungs were fixed in Bouin's solution (Fig. 4C), and the metastases quantified (Fig. 4D). No statistical significant differences were found between the two groups.

Almost all MMTV-PyMT mice developed lung metastases, and all died of lung metastasis within several weeks of development. To observe the effect of MOGAT2 depletion on survival, we collected the survival 
time of the two groups of mice and found that MOGAT2 depletion did not significantly influence overall survival $(P=0.977)($ Fig. $5 A)$.

\section{A high fat diet accelerates MMTV-PyMT mammary tumor incidence and growth regardless of MOGAT2 depletion}

Previous studies $(13,14,20)$ reported that MOGAT2 ablation protects mice from high-fat diet $(60 \%$ of calories from fat) induced obesity. We investigated the effect of MOGAT2's depletion on mammary tumorigenesis of MMTV-PyMT mice fed with a relatively high fat diet (37\% of calories from fat). Female Mogat2 $^{+/+}$PyMT and Mogat2-/-PyMT mice were produced by mating MMTV-PyMT males with Mogat2-1

- females. Both of them were fed with a relatively high fat diet, commencing at 5 weeks old which continued for 12 weeks. Compared with normal diet-fed Mogat2 ${ }^{+/+}$PyMT mice, a relatively high fat diet significantly accelerated mammary tumor incidence and growth regardless of MOGAT2 depletion (Fig. 5B and $5 \mathrm{C})$.

\section{DGAT1 and DGAT2 act as functional substitutes in MOGAT2-/-PyMT mice}

To investigate the mechanism of functional cover-up upon MOGAT2 depletion in mice, we collected the mammary tumors, stomach, small intestine and colorectal tissues of the mice with or without MOGAT2 depletion, and examined the RNA levels of MOGAT1, MOGAT2, DGAT1 and DGAT2 by qPCR (Fig. 6). Compared with the high fat diet-fed MOGAT $2^{+/+}$PyMT mice, a significant reduction in MOGAT2 was evident in the high fat diet-fed MOGAT2 ${ }^{-/-}$PyMT mice, while MOGAT1 expression was unaltered (Fig. 6B). However, DGAT1 and DGAT2 were significantly upregulated in the gastrointestinal tract in Mogat2-/ -PyMT mice (Fig. 6C and D), suggesting that both DGAT1 and DGAT2 play some role along with MOGAT2 which promote obesity.

\section{Discussion}

Previous studies $(13,14)$ showed that MOGAT2 ablation protects mice from high fat diet induced obesity. In our study, a relatively high fat diet (37\% of calories from fat) promoted obesity and subsequent mammary tumor formation and metastasis regardless of Mogat2 depletion. This discrepancy however, may be the result of a lower amount of fat in the food: $60 \%$ versus $37 \%$.

In terms of dietary habits, most countries in Asia have a relatively low total fat intake ( $<30 \%$ total energy, TE), such as $20 \%$ TE for China (21), 22.5\% TE for India (22), 21.1\% TE for South Korea (23) and 23.3$26.3 \%$ TE for Japan (24), whereas, most western countries have a relatively higher total fat intake (>30\% TE), with $34 \%$ TE for the USA (25), 37.6\% TE for Germany (26) and 38.2\% TE for France (27). A systematic review of data from 40 countries found that the total fat intake ranged from 11.1 to $46.2 \% \mathrm{TE}$ (25). Therefore, the relatively high fat diet (37\% of calories from fat) used in our study is more in line with 
human dietary habits compared with the higher fat diet (60\% of calories from fat) used in other studies $(4,13,14)$.

Our findings about the upregulation of Dgat1 and Dgat2 expression upon Mogat2 depletion suggest that genetic depletion of MOGAT2 alone is insufficient in preventing obesity and mammary tumor formation. DGAT1 and DGAT2 are abundantly expressed in small intestine $(28,29)$ and mediate the final step in TAG re-synthesis during dietary fat absorption. Previous studies have shown that DGAT1 (15) and DGAT2 have the capacity to acylate MAG to form DAG (16). These results imply that DGAT1 and DGAT2 can act as functional substitutes in Mogat2-/-PyMT mice.

\section{Conclusions}

In summary, elevated expression of MOGAT2 in triple negative breast cancer correlates with poorer patient overall survival. With the compensation of DGAT1 and DGAT2, MOGAT2 depletion alone, cannot prevent fat food-induced obesity, and has no effect on mammary tumor initiation, growth or metastasis. Targeting multiple key enzymes in fat metabolism might generate better outcomes in controlling obesity and probably in inhibiting mammary tumor development as well.

\section{Abbreviations}

\section{MOGAT2}

monoacylglycerol O-acyltransferase 2

Dgat1

diacylglycerol 0-acyltransferases 1

Dgat2

diacylglycerol 0-acyltransferases 2

MOGAT

monoacylglycerol acyltransferase

DAG

diacylglycerol

DGAT

diacylglycerol acyltransferase

TAG

re-synthesize triacylglycerol.

\section{Declarations}

\section{Ethics approval and consent to participate}

This animal study was approved by the Sun Yat-sen University Cancer Center's Institutional Animal Care and Usage Committee (L102012017000G). All human tissue samples were obtained with patient consent 
and the approval of the Institutional

Clinical Ethics Review Board at SYSUCC.

\section{Consent for publication}

Not applicable

\section{Availability of data and materials}

The datasets generated during and/or analyzed during the current study are

available from the corresponding author on reasonable request.

\section{Competing interests}

The authors declare that they have no competing interests.

\section{Funding}

This work was supported by grants from the National Natural Science Foundation of

China (No. 82073220), the Foundation of China Postdoctoral Science (No. 2019 M663297 to Y.M.).

\section{Authors' contributions}

Conception and design: Chao-Nan Qian

Development of methodology: Li-Xia Peng

Acquisition of data (provided animals, acquired and managed patients, provided facilities, etc.): Yan Mei, Jia-Bin Lu, Xiao-Dong He

Analysis and interpretation of data (e.g., statistical analysis, biostatistics, computational analysis): Yan Mei, Jing Wang, Guan-Ming Lu

Writing, review, and/or revision of the manuscript: Yan Mei

Administrative, technical, or material support (i.e., reporting or organizing data, constructing databases): Yan Mei, Jia-Bin Lu

Study supervision: Chao-Nan Qian

\section{Acknowledgements}

The authors thank Dr. Xiao-Dong He at the Guangdong Pharmaceutical University for her assistance with mouse feeding. 


\section{References}

1. Picon-Ruiz M, Morata-Tarifa C, Valle-Goffin JJ, Friedman ER, Slingerland JM. Obesity and adverse breast cancer risk and outcome: Mechanistic insights and strategies for intervention. CA Cancer J Clin. 2017;67(5):378-97.

2. Heer E, Harper A, Escandor N, Sung H, McCormack V, Fidler-Benaoudia MM. Global burden and trends in premenopausal and postmenopausal breast cancer: a population-based study. The Lancet Global health. 2020;8(8):e1027-e37.

3. Teras LR, Patel AV, Wang M, Yaun SS, Anderson K, Brathwaite R, et al. Sustained Weight Loss and Risk of Breast Cancer in Women 50 Years and Older: A Pooled Analysis of Prospective Data. J Natl Cancer Inst. 2020;112(9):929-37.

4. Nelson DW, Gao Y, Spencer NM, Banh T, Yen CL. Deficiency of MGAT2 increases energy expenditure without high-fat feeding and protects genetically obese mice from excessive weight gain. J Lipid Res. 2011;52(9):1723-32.

5. Ros E. Intestinal absorption of triglyceride and cholesterol. Dietary and pharmacological inhibition to reduce cardiovascular risk. Atherosclerosis. 2000;151(2):357-79.

6. Kayden HJ, Senior JR, Mattson FH. The monoglyceride pathway of fat absorption in man. J Clin Invest. 1967;46(11):1695-703.

7. Phan CT, Tso P. Intestinal lipid absorption and transport. Front Biosci. 2001;6:D299-319.

8. Mattson FH, Volpenhein RA. The Digestion and Absorption of Triglycerides. J Biol Chem. 1964;239:2772-7.

9. Yen CL, Farese RV. Jr. MGAT2, a monoacylglycerol acyltransferase expressed in the small intestine. J Biol Chem. 2003;278(20):18532-7.

10. Yen CL, Stone SJ, Cases S, Zhou P, Farese RV Jr. Identification of a gene encoding MGAT1, a monoacylglycerol acyltransferase. Proc Natl Acad Sci U S A. 2002;99(13):8512-7.

11. Cheng D, Nelson TC, Chen J, Walker SG, Wardwell-Swanson J, Meegalla R, et al. Identification of acyl coenzyme A:monoacylglycerol acyltransferase 3 , an intestinal specific enzyme implicated in dietary fat absorption. J Biol Chem. 2003;278(16):13611-4.

12. Cao J, Hawkins E, Brozinick J, Liu X, Zhang H, Burn P, et al. A predominant role of acylCoA:monoacylglycerol acyltransferase-2 in dietary fat absorption implicated by tissue distribution, subcellular localization, and up-regulation by high fat diet. J Biol Chem. 2004;279(18):18878-86.

13. Yen CL, Cheong ML, Grueter C, Zhou P, Moriwaki J, Wong JS, et al. Deficiency of the intestinal enzyme acyl CoA:monoacylglycerol acyltransferase-2 protects mice from metabolic disorders induced by high-fat feeding. Nat Med. 2009;15(4):442-6.

14. Nelson DW, Gao Y, Yen MI, Yen CL. Intestine-specific deletion of acyl-CoA:monoacylglycerol acyltransferase (MGAT) 2 protects mice from diet-induced obesity and glucose intolerance. J Biol Chem. 2014;289(25):17338-49. 
15. Yen CL, Monetti M, Burri BJ, Farese RV. Jr. The triacylglycerol synthesis enzyme DGAT1 also catalyzes the synthesis of diacylglycerols, waxes, and retinyl esters. J Lipid Res. 2005;46(7):150211.

16. Cheng D, Iqbal J, Devenny J, Chu CH, Chen L, Dong J, et al. Acylation of acylglycerols by acyl coenzyme A:diacylglycerol acyltransferase 1 (DGAT1). Functional importance of DGAT1 in the intestinal fat absorption. J Biol Chem. 2008;283(44):29802-11.

17. Cao J, Cheng L, Shi Y. Catalytic properties of MGAT3, a putative triacylgycerol synthase. J Lipid Res. 2007;48(3):583-91.

18. de Assis S, Warri A, Cruz MI, Hilakivi-Clarke L. Changes in mammary gland morphology and breast cancer risk in rats. Journal of visualized experiments: JoVE. 2010(44).

19. Spandidos A, Wang X, Wang H, Seed B. PrimerBank: a resource of human and mouse PCR primer pairs for gene expression detection and quantification. Nucleic acids research. 2010;38(Database issue):D792-9.

20. Tsuchida T, Fukuda S, Aoyama H, Taniuchi N, Ishihara T, Ohashi N, et al. MGAT2 deficiency ameliorates high-fat diet-induced obesity and insulin resistance by inhibiting intestinal fat absorption in mice. Lipids Health Dis. 2012;11:75.

21. Ueshima H, Stamler J, Elliott P, Chan Q, Brown IJ, Carnethon MR, et al. Food omega-3 fatty acid intake of individuals (total, linolenic acid, long-chain) and their blood pressure: INTERMAP study. Hypertension. 2007;50(2):313-9.

22. Sudha V, Radhika G, Sathya RM, Ganesan A, Mohan V. Reproducibility and validity of an intervieweradministered semi-quantitative food frequency questionnaire to assess dietary intake of urban adults in southern India. Int J Food Sci Nutr. 2006;57(7-8):481-93.

23. Kim J, Kim YJ, Ahn YO, Paik HY, Ahn Y, Tokudome Y, et al. Contribution of specific foods to fat, fatty acids, and cholesterol in the development of a food frequency questionnaire in Koreans. Asia Pac $J$ Clin Nutr. 2004;13(3):265-72.

24. Saito A, Imai S, Htun NC, Okada E, Yoshita K, Yoshiike N, et al. The trends in total energy, macronutrients and sodium intake among Japanese: findings from the 1995-2016 National Health and Nutrition Survey. Br J Nutr. 2018;120(4):424-34.

25. Harika RK, Eilander A, Alssema M, Osendarp SJ, Zock PL. Intake of fatty acids in general populations worldwide does not meet dietary recommendations to prevent coronary heart disease: a systematic review of data from 40 countries. Ann Nutr Metab. 2013;63(3):229-38.

26. Linseisen J, Schulze MB, Saadatian-Elahi M, Kroke A, Miller AB, Boeing H. Quantity and quality of dietary fat, carbohydrate, and fiber intake in the German EPIC cohorts. Ann Nutr Metab. 2003;47(1):37-46.

27. Elmadfa I. European Nutrition and Health Report 2009. Preface Forum Nutr. 2009;62:vii-viii.

28. Cases S, Smith SJ, Zheng YW, Myers HM, Lear SR, Sande E, et al. Identification of a gene encoding an acyl CoA:diacylglycerol acyltransferase, a key enzyme in triacylglycerol synthesis. Proc Natl Acad Sci U S A. 1998;95(22):13018-23. 
29. Cases S, Stone SJ, Zhou P, Yen E, Tow B, Lardizabal KD, et al. Cloning of DGAT2, a second mammalian diacylglycerol acyltransferase, and related family members. J Biol Chem. 2001;276(42):38870-6.

\section{Figures}
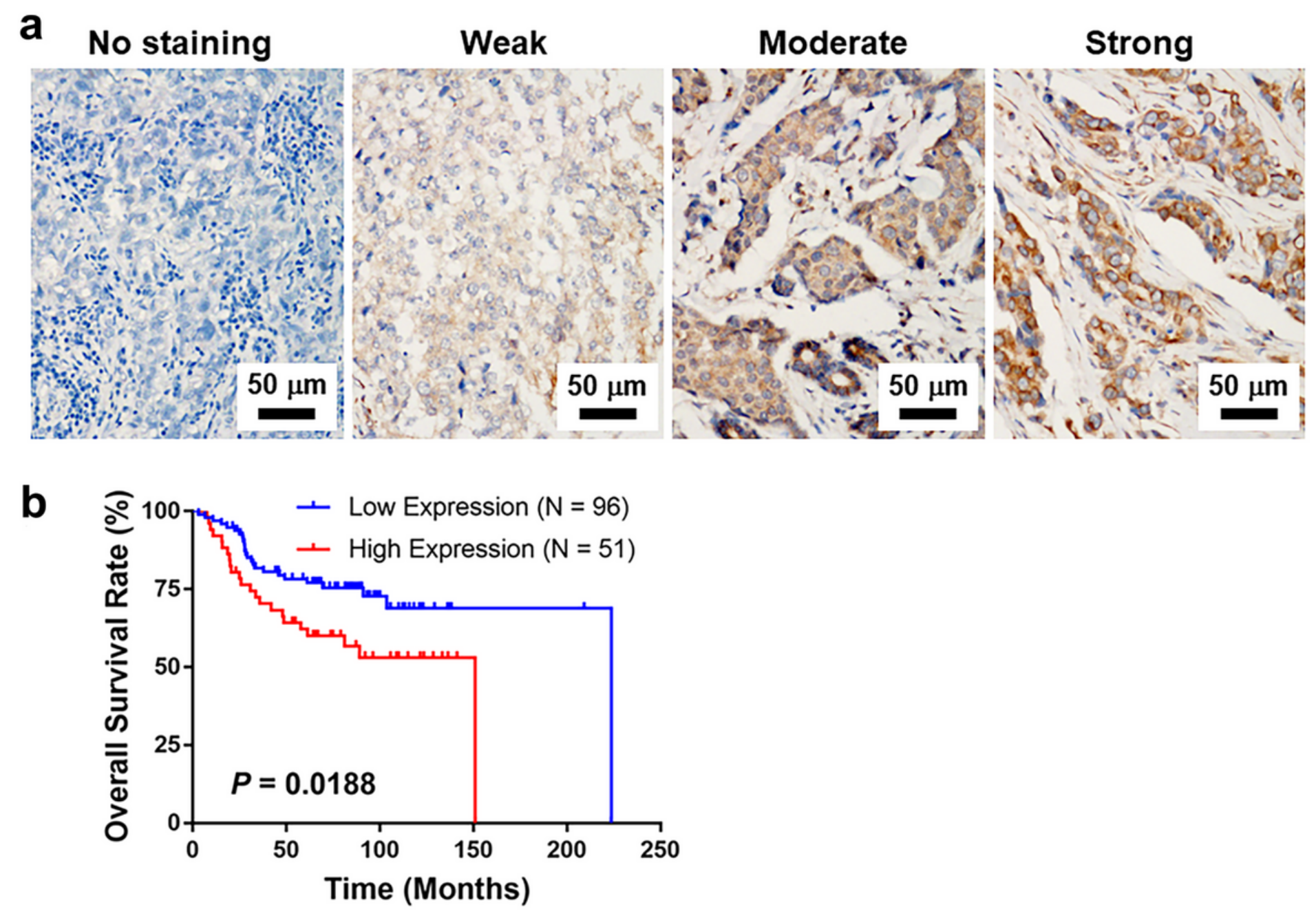

Figure 1

Primary tumor MOGAT2 expression level negatively correlates with poorer breast cancer patient survival. (a) Eight tissue arrays consisting of 147 triple-negative breast cancer samples were constructed and immunohistochemically stained with a MOGAT2 antibody, followed by an assessment including the percentage of positively stained tumor cells and the intensity of staining on a 13-point scale. (b) Survival analyses showed that in the group with elevated MOGAT2 expression in the tumor, breast cancer patients had a poorer overall survival (OS) probability. 


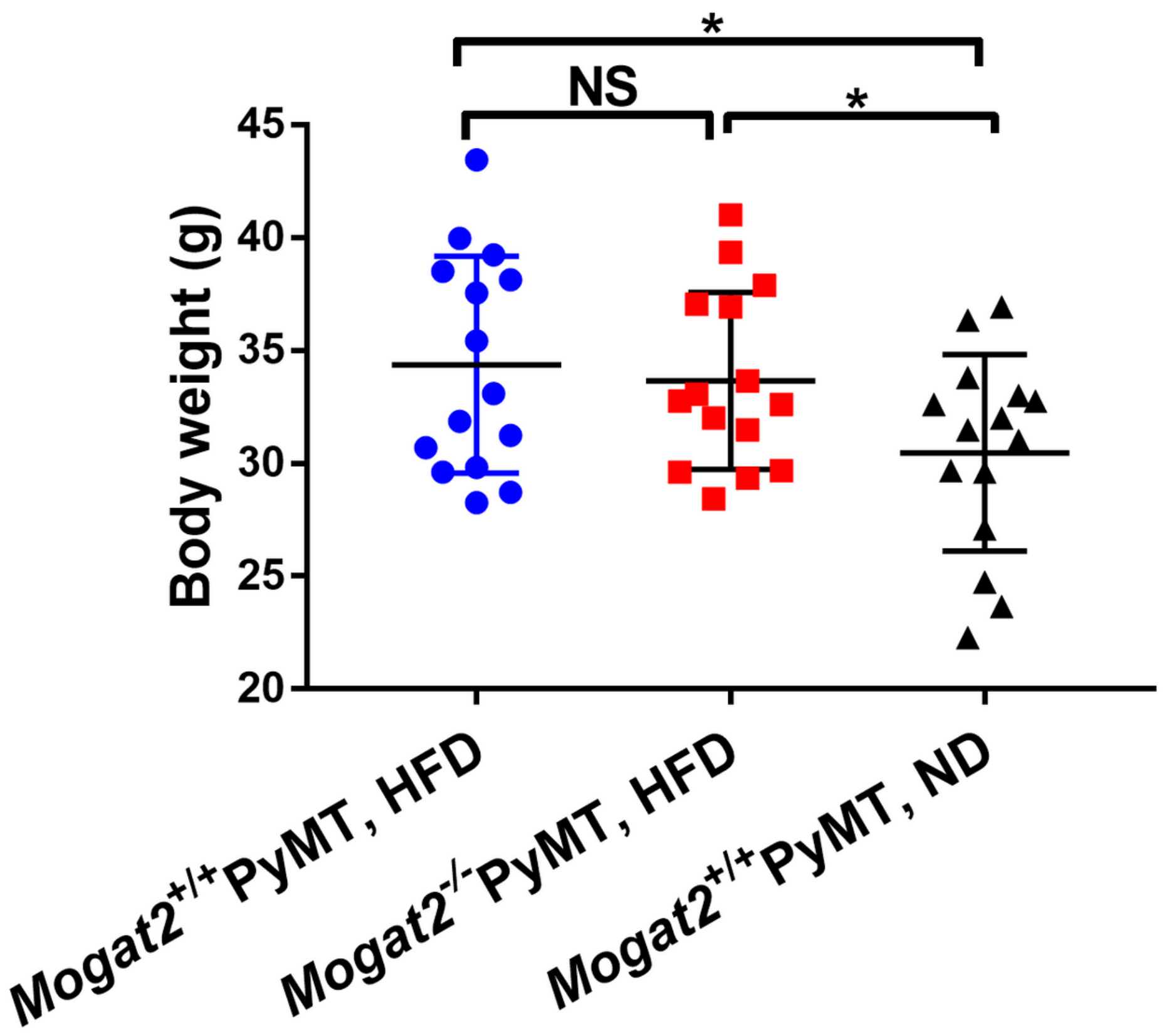

Figure 2

A relatively high fat diet promotes obesity regardless of Mogat2 depletion Body weight was determined in Mogat2+/+PyMT ( $n=15)$ and Mogat2-/-PyMT $(n=15)$ mice fed with a relatively high fat diet, as well as in the control group of Mogat2+/+PyMT $(n=15)$ fed with a normal diet. Increments in body weight were found to be independent of the Mogat2 gene. NS = non-significant. 
a
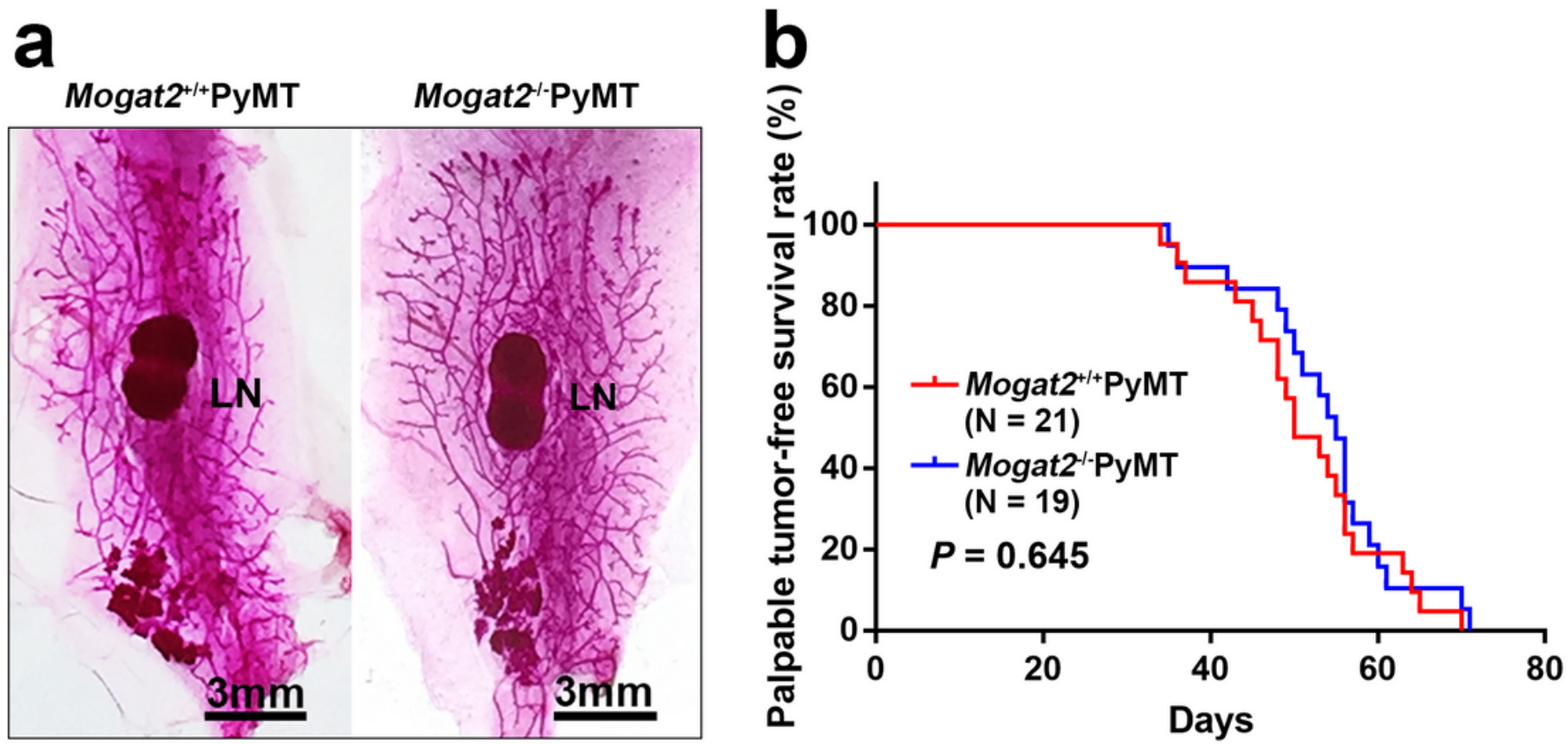

Figure 3

Mammary tumor initiation is not delayed by Mogat2 gene depletion. (a) Whole mount images of the fourth pair of mammary glands from Mogat2+/+PyMT and Mogat2-/-PyMT mice at 5 weeks old. LN = lymph node. (b) Kaplan Meier plots of the tumor latency of Mogat2+/+PyMT ( $n=19)$ and Mogat2-/ -PyMT $(n=21)$ mice. Data were analyzed by the log rank test. All mice were fed a normal diet $(10 \%$ of calories from fat). 


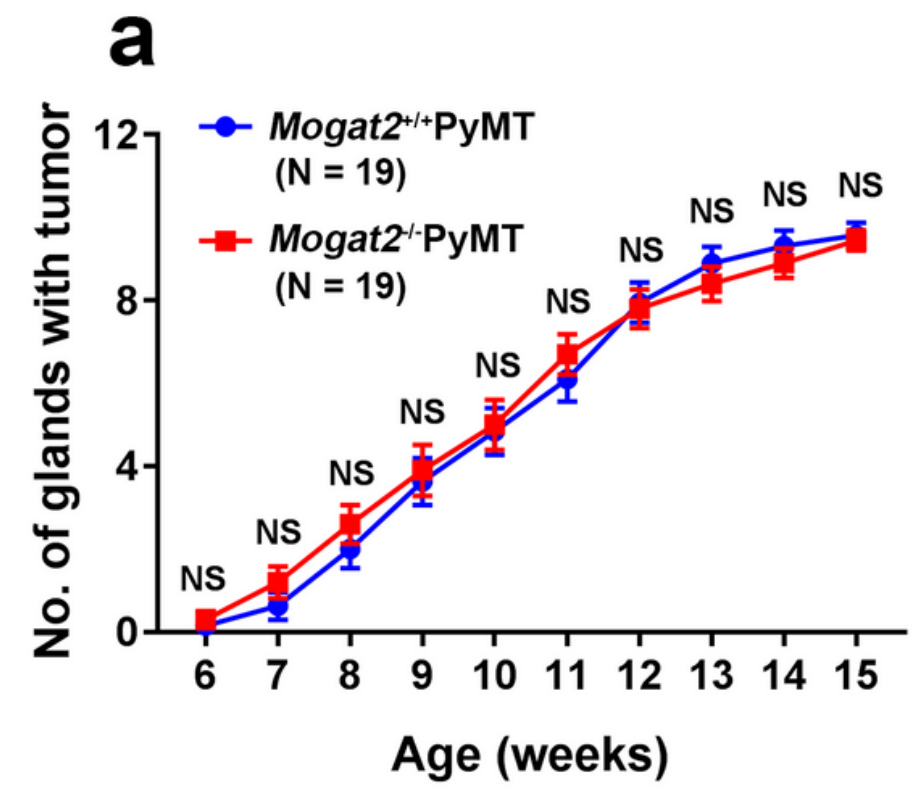

C

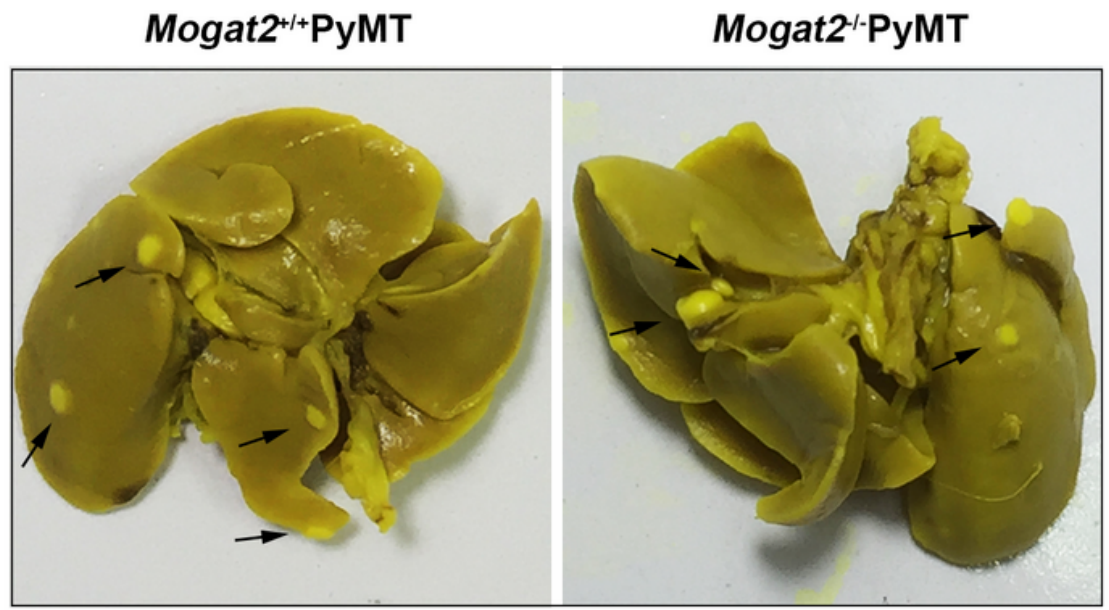

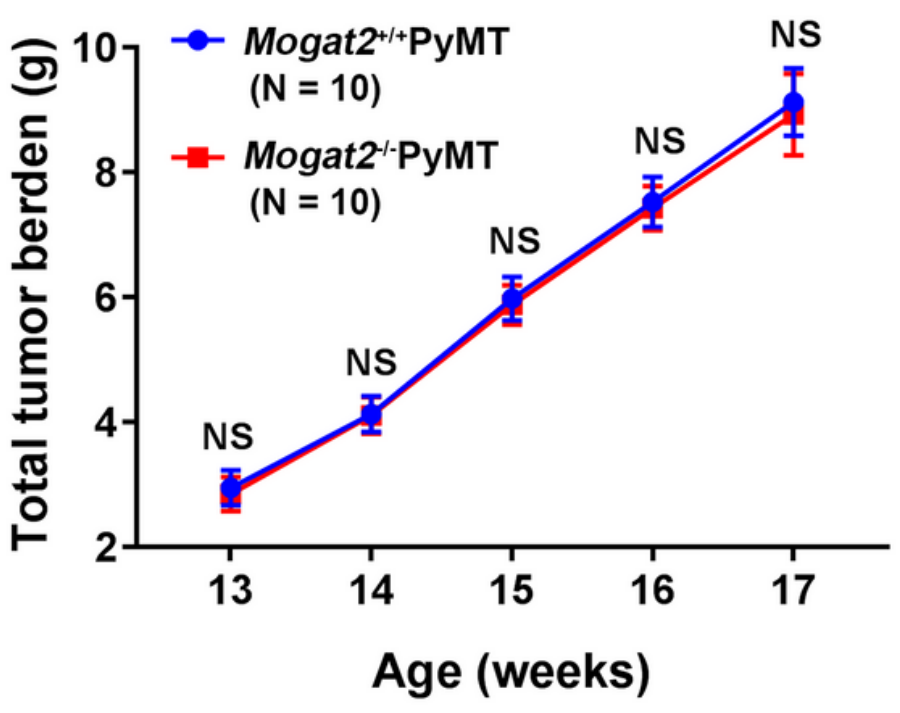

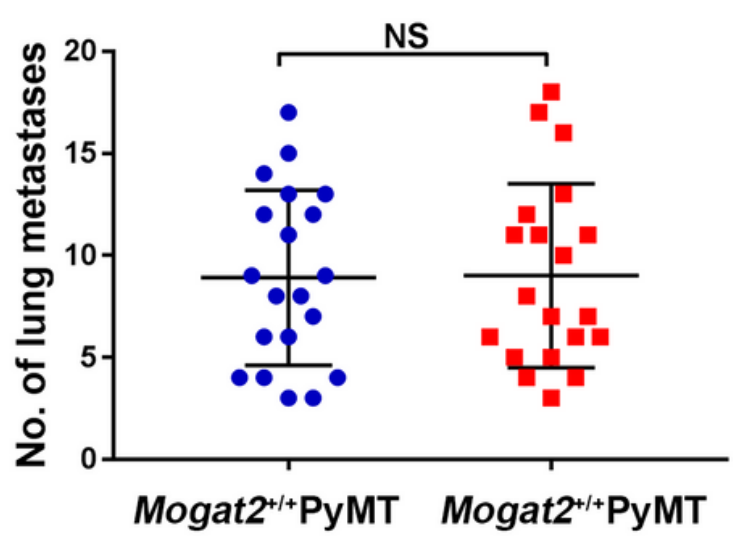

Figure 4

Mammary tumor progression is not inhibited by Mogat2 gene depletion. (a) The number of mammary glands with palpable tumors for Mogat2+/+PyMT $(n=19)$ and Mogat2-/-PyMT $(n=19)$ mice. Data shown are the number of tumor-bearing glands per mouse. Mammary tumors were monitored by weekly palpation. (b) Total tumor volume for Mogat2+/+PyMT $(n=10)$ and Mogat2-/-PyMT ( $n=10)$ mice. (c-d) Lungs from Mogat2+/+PyMT $(n=20)$ and Mogat2-/-PyMT $(n=20)$ mice were stained with Bouin's solution to identify metastases. Metastatic lesions are indicated by the arrows. (c) The data represents the mean total number of metastases \pm SEM from both groups at 15 weeks old (d) Data represents the mean \pm SEM, NS = non-significant. All mice were fed a normal diet. 

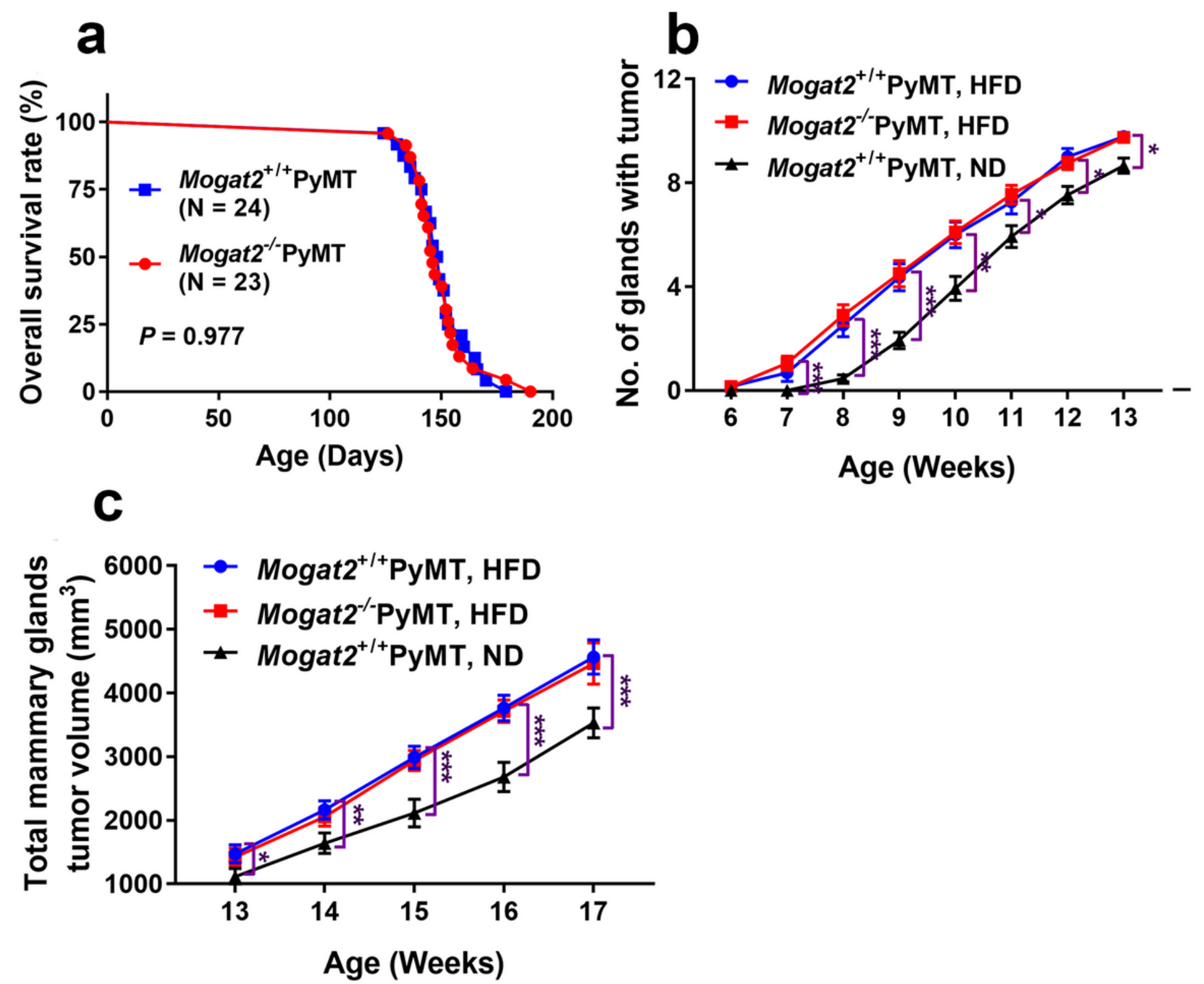

Figure 5

Mammary tumor progression is accelerated by a high fat diet regardless of Mogat2 gene depletion.

(a)Kaplan-Meier curves showing the overall survival of Mogat2+/+PyMT ( $n=24)$ and Mogat2-/-PyMT $(n=24)$ mice. Data were analyzed by the log rank test. The mice were fed on a normal diet. (b)The appearance of mammary tumors in Mogat2+/+PyMT $(n=19)$ and Mogat2-/-PyMT $(n=20)$ mice fed with a high fat diet (37\% of calories from fat), and Mogat2+/+PyMT ( $n=15)$ fed on a normal diet were monitored by weekly palpation and are represented on Kaplan-Meier curves. (c) The tumor volume of all mammary glands were determined in Mogat2+/+PyMT $(n=10)$ and Mogat2-/-PyMT $(n=10)$ mice fed on a high fat diet, as well as Mogat2+/+PyMT ( $n=10)$ mice fed on a normal diet were measured weekly at 13-17 weeks old. Data represent the mean \pm SEM. NS, non-significant; HFD, high-fat diet; ND, normal diet. $\star, P<0.05$. **, $\mathrm{P}<0.01$. ***, $\mathrm{P}<0.001$. 

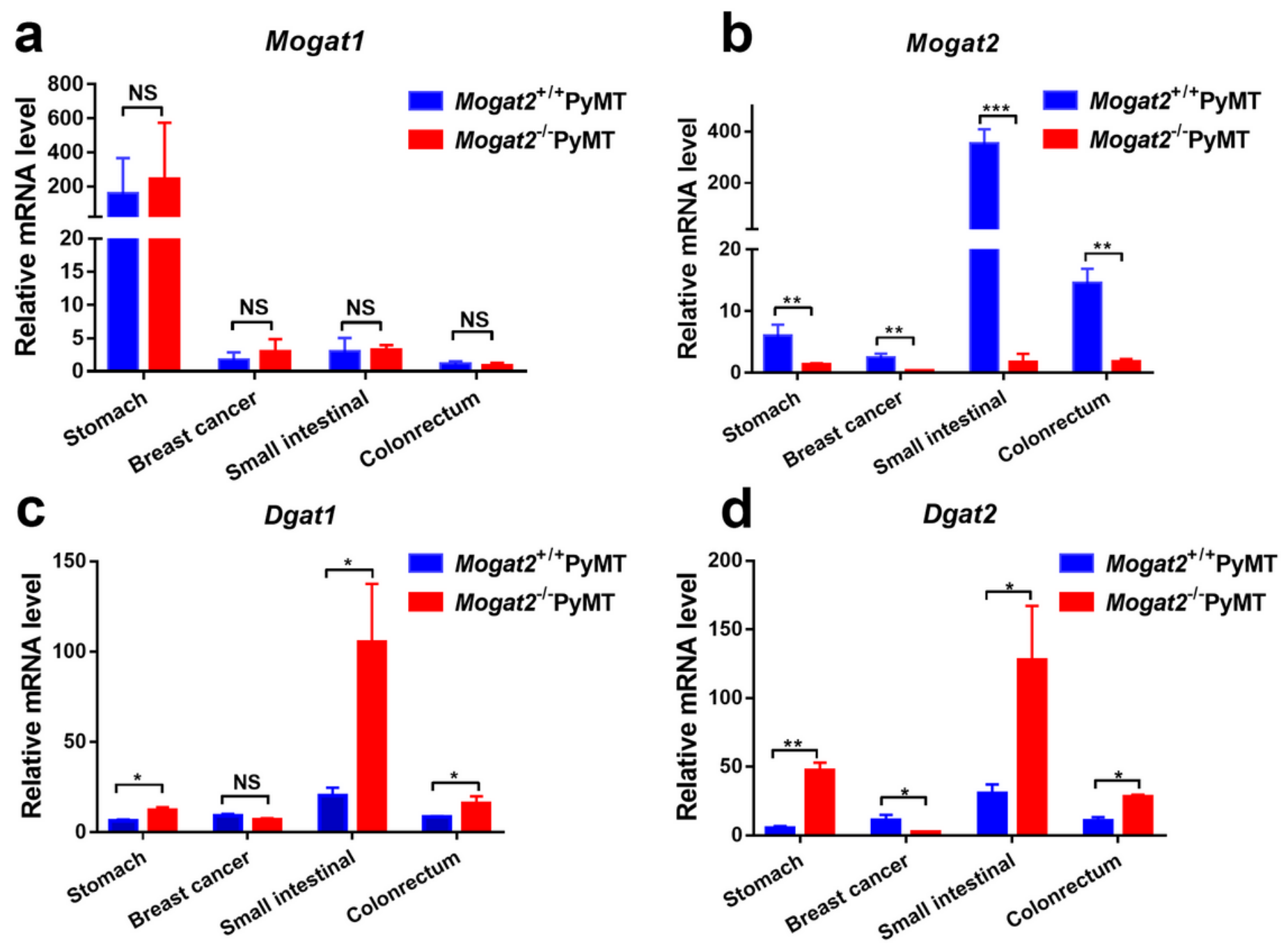

Figure 6

Depletion of the Mogat2 gene results in compensational overexpression of Dgat1 and Dgat2 genes. (a) Mogat1 gene mRNA was detected by qPCR in breast cancer, stomach, small intestine and colorectal tissues from Mogat2+/+PyMT $(n=6)$ and Mogat2-/-PyMT $(n=6)$ mice. As expected, Mogat1 gene expression was unaltered upon Mogat2 depletion. (b) In the same tissues, the Mogat2 expression level was suppressed in all the analyzed organs. (c) To compensate, Dgat1's mRNA level was upregulated in the gastrointestinal system, especially in the small intestine, which is the major organ for fat absorption. (d) The level of Dgat2 mRNA had a similar overexpression pattern with that of Dgat1. Data represent the mean $\pm S E M, N S=$ non-significant, $* P<0.05, * \star P<0.01$ and ${ }^{\star *} * \mathrm{P}<0.001$. 